MATHEMATICS OF COMPUTATION

Volume 73 , Number 246 , Pages $525-539$

S 0025-5718(03)01566-7

Article electronically published on July 14, 2003

\title{
A MULTILEVEL SUCCESSIVE ITERATION METHOD FOR NONLINEAR ELLIPTIC PROBLEMS
}

\author{
YUNQING HUANG, ZHONGCI SHI, TAO TANG, AND WEIMIN XUE
}

\begin{abstract}
In this paper, a multilevel successive iteration method for solving nonlinear elliptic problems is proposed by combining a multilevel linearization technique and the cascadic multigrid approach. The error analysis and the complexity analysis for the proposed method are carried out based on the two-grid theory and its multilevel extension. A superconvergence result for the multilevel linearization algorithm is established, which, besides being interesting for its own sake, enables us to obtain the error estimates for the multilevel successive iteration method. The optimal complexity is established for nonlinear elliptic problems in 2-D provided that the number of grid levels is fixed.
\end{abstract}

\section{INTRODUCTION}

The multigrid method (MG) has been shown to be one of the most efficient techniques for solving partial differential equations and has been studied by many researchers, see, e.g., Brandt [6] and Hackbusch [12] and the references cited therein. The nested version of the multigrid method or the so-called full multigrid method (FMG) can yield the optimal order of operations $\mathcal{O}(N)$ in obtaining the approximate solution with the accuracy of discretization. In contrast with FMG, Deuflhard 9 proposed a cascadic iteration algorithm which employs nested iterations using the conjugate gradient $(\mathrm{CG})$ method or the preconditioned conjugate gradient (PCG) method instead of using MG at each level. Some adaptive strategies are also proposed, see, e.g., 9, 10. The efficiency of the cascadic algorithm has been demonstrated numerically in [9, 10, and the comparison is made with the numerical results obtained by using the multilevel preconditioner of Bramble, Pasciak and $\mathrm{Xu}[5$ and the hierarchical preconditioner of Yserentant [27]. The main feature of the cascadic iteration is coarse-grid-correction free, and as a result it can be viewed as a one-way multigrid method. Since the cascadic iteration never goes back to the coarse grids, the error associated with the coarse grids is of relatively low frequencies when the iteration reaches the fine grid, which is very hard to reduce by using a conventional smoother. Hence, the cascadic algorithm has to solve the underlying problems on each level to the same accuracy required for the final level, while FMG solves the underlying problems on each level to the discretization accuracy required for the current level. This implies that the cascadic algorithm may require a large amount of computational time on coarse grids. From the theoretical point of view,

Received by the editor June 6, 2000 and, in revised form, October 12, 2002.

2000 Mathematics Subject Classification. Primary 65F10, 65N30, 65N55.

Key words and phrases. Multigrid method, cascadic algorithm, finite element method, nonlinear elliptic problem, error estimate, complexity. 
another disadvantage of the cascadic iteration algorithm is that the optimal error estimate can be obtained only under the energy norm.

On the other hand, the main advantage of the cascadic algorithm is its simplicity. It is also efficient for a large class of problems, as demonstrated in [4, 9, 10]. Let the index of the final level be $J$ and that of the current level be $j$. The iteration number on each grid level can be determined a priori or a posteriori, which in general depends only on the difference $J-j$, but not on the spatial dimensions. It is the independence of the spatial dimension that yields better efficiency in higher dimensions. In recent years, there have been several analyses and applications of the cascadic iteration algorithm, e.g., Shi and $\mathrm{Xu}$ 19, 20, 21 applied the cascadic multigrid technique to elliptic problems with nonconforming elements, to the plate bending problem, and to parabolic problems; and Braess and Dehmen [4] applied the cascadic algorithm to the Stokes equations. On the theoretical side, Shaidurov 18. obtained the optimal complexity in $H^{2}$ for the cascadic algorithm with CG as a smoother. Bornemann and Deuflhard [2] analyzed the cascadic algorithm for some general smoothers such as the damped Jacobi, the Gauss-Seidel, etc., under the weaker $H^{1+\alpha}$-regularity assumption with $0<\alpha \leq 1$.

The main objective of this paper is to study a multilevel successive iteration algorithm for solving nonlinear elliptic equations. In obtaining the algorithm, a multilevel linearization approach and a cascadic multigrid iteration technique are employed. The error analysis and complexity analysis will be carried out by using the theory for the two-grid method which was first introduced by Xu 24, 25] in approximating nonsymmetric indefinite nonlinear problems. It is based on the facts that the low frequencies are governed by some nonlinear nonsymmetric indefinite operators on the coarse grid and the related high frequencies are governed by some linear symmetric positive definite (SPD) operators on the fine grid. Therefore, we can solve a rather complicated problem on the coarse grids, and then solve an easier problem (linear, SPD) on the fine grid as a correction. If the solution on a coarse grid is sufficiently accurate, then the correction (i.e., the difference between a finer grid solution and the coarse grid solution) can be easily obtained by using simple smoothers. It will be shown that on coarse grids only a fixed number of smoothing iterations are needed. Moreover, the iteration number depends not on the meshsize of the coarse grid but on the number of the refinements used. By extending the two-grid theory to the multigrid case, a multilevel successive iteration algorithm can be proposed to solve a class of nonlinear finite element equations. We note that the idea of using the successive iterations to provide reasonable initial values for linear problems can be traced back to Huang and Liu [14. However, there had been no theoretical justification until the cascadic multigrid iteration algorithm appeared.

We now state some notation and conventions for later use. Let $\Omega \subset R^{d}$ be a bounded convex polygonal domain of dimension $d$, and let $W^{k, p}(\Omega)$ be Sobolev space equipped with the $L^{p}$ norm and semi-norm:

$$
\|u\|_{k, p, \Omega}=\left(\int_{\Omega} \sum_{|\alpha| \leq k}\left|D^{\alpha} u\right|^{p}\right)^{\frac{1}{p}}, \quad|u|_{k, p, \Omega}=\left(\int_{\Omega} \sum_{|\alpha|=k}\left|D^{\alpha} u\right|^{p}\right)^{\frac{1}{p}},
$$

where $\alpha=\left(\alpha_{1}, \ldots, \alpha_{d}\right)$ is a multi-index. When $p=2$ we $\operatorname{denote} W^{k, 2}(\Omega)$ by $H^{k}(\Omega)$ and omit the index $p$ in the norm notation. $H_{0}^{1}(\Omega)$ consists of functions in $H^{1}(\Omega)$ that vanish on the boundary $\partial \Omega$. $H^{-1}(\Omega)$ is the dual of $H_{0}^{1}(\Omega)$. We shall use the 
notation $\lesssim, \gtrsim, \equiv$ as in Xu [26]: when we write $x_{1} \lesssim y_{1}, x_{2} \gtrsim y_{2}, x_{3} \equiv y_{3}$, it means that there exist constants $c_{1}, c_{2}, c_{3}, C_{3}$ such that

$$
x_{1} \leq c_{1} y_{1}, \quad x_{2} \geq c_{2} y_{2}, \quad c_{3} x_{3} \leq y_{3} \leq C_{3} x_{3},
$$

where the $c_{i}$ 's and $C_{3}$ are constants independent of the mesh level $j$ and mesh size $h_{j}$. These constants may be different at different places. Some special constants will be defined later. Throughout this paper, the Einstein summation convention is used: summation is taken over repeated indices. For example, $a_{i j} b_{i}$ denotes $\sum_{i=1}^{n} a_{i j} b_{i}$.

The rest of the paper is organized as follows. In Section 2 some preliminaries relevant to our error and complexity analysis will be provided. In particular, we will briefly review and study the two-grid method and the multilevel linearization technique. With these preparations, a multilevel successive cascadic iteration algorithm will be proposed and analyzed in Section 3

\section{Preliminaries}

Let us consider the following second-order nonlinear elliptic problem:

$$
\left\{\begin{aligned}
L(u)=-\partial_{i}\left(a_{i}(x, \nabla u)\right) & =f(x) & & \text { in } \Omega, \\
u & =0 & & \text { on } \partial \Omega .
\end{aligned}\right.
$$

We assume that $a_{i}(x, y): \bar{\Omega} \times \mathcal{R}^{d} \rightarrow \mathcal{R}^{1}$ is smooth and 2.1) has a unique and nonsingular solution $u \in H_{0}^{1}(\Omega) \cap W^{2, d+\epsilon}$ for some $\epsilon>0$. The linearized operator $L^{\prime}$ of $L$ at $w$ is defined by the Fréchet derivative as

$$
L^{\prime}(w) \phi=-\partial_{i}\left(a_{i j}(x, \nabla w) \partial_{j} \phi\right)
$$

where

$$
a_{i j}(x, \nabla w)=\frac{\partial a_{i}(x, \nabla w)}{\partial y_{j}} .
$$

Assume that $a_{i j}(x, \nabla w)$ is SPD for $w$ in a neighborhood of the solution $u$ for (2.1), i.e., there exist two constants $\alpha_{0}$ and $K$ such that, $\forall \xi \in \mathcal{R}^{d}$,

$$
a_{i j}(x, \nabla w) \xi_{i} \xi_{j} \geq \alpha_{0}|\xi|^{2} \quad \forall w \in \mathcal{B}_{K},
$$

where

$$
\mathcal{B}_{K}=\left\{w \in W^{1, \infty}:\|w-u\|_{1, \infty} \leq K\right\}
$$

Let

$$
A(u, v):=\left(a_{i}(x, \nabla u), \partial_{i} v\right)
$$

and

$$
A^{\prime}(w ; \phi, v):=\left(L^{\prime}(w) \phi, v\right)=\left(a_{i j}(x, \nabla w) \partial_{j} \phi, \partial_{i} v\right) .
$$

Assume that $A^{\prime}$ is bounded in a neighborhood of $u$ in the following sense:

$$
\left|A^{\prime}(w, \phi, v)\right| \leq M\|\phi\|_{1}\|v\|_{1} \quad \forall \phi, v \in H_{0}^{1}(\Omega), w \in \mathcal{B}_{K} .
$$

Then the weak solution $u \in H_{0}^{1}(\Omega)$ for (2.1) is defined by the following equation:

$$
A(u, v)=(f, v) \quad \forall v \in H_{0}^{1}(\Omega)
$$


where $(f, v)$ is the standard inner product in $\Omega$. Let $V^{h} \subset H_{0}^{1}(\Omega)$ be a finite element space. The finite element approximation $u_{h} \in V^{h}$ for the above problem is then defined by

$$
A\left(u_{h}, v\right)=(f, v) \quad \forall v \in V^{h} .
$$

For any $u, v, w \in H_{0}^{1}$, set $\eta(t)=A(w+t(u-w), v)$. Since

$$
\eta(1)-\eta(0)=\eta^{\prime}(0)+\int_{0}^{1} \eta^{\prime \prime}(t)(1-t) d t,
$$

we obtain the equality

$$
A(u, v)-A(w, v)=A^{\prime}(w ; u-w, v)+R(w, u, v),
$$

where the last term satisfies

$$
|R(w, u, v)| \lesssim\|u-w\|_{1,2 p}^{2}\|v\|_{1, p^{\prime}}, \quad \frac{1}{p}+\frac{1}{p^{\prime}}=1 .
$$

The last estimate is obtained by calculating $\eta^{\prime \prime}(t)$ directly and by using a Höldertype inequality, see also 25]. Replacing $(w, u)$ in (2.10) by the solution $u$ of (2.8) and $u_{h}$ of (2.9) gives

$$
A^{\prime}\left(u ; u-u_{h}, v\right)=R\left(u, u_{h}, v\right) \quad \forall v \in V^{h}
$$

or

$$
A^{\prime}\left(u_{h} ; u_{h}-u, v\right)=R\left(u_{h}, u, v\right) \quad \forall v \in V^{h} .
$$

The existence and uniqueness for the finite element approximation (2.9) and its error estimates can be found in Frehse and Rannacher [11, Rannacher [15], Xu [24], and Chen and Huang [8].

Lemma 2.1 (Xu [24]). If $u \in W^{2, d+\epsilon}(\Omega)$ and $u_{h} \in V^{h}$ are the solution of (2.8) and (2.9) respectively, then the following estimates hold:

$$
\begin{array}{lll}
\left\|u-u_{h}\right\|_{1, p} & \lesssim h & \text { if } u \in W^{2, p}, 2 \leq p \leq \infty \\
\left\|u-u_{h}\right\|_{0, p} & \lesssim h^{2} & \text { if } u \in W^{2, p}, 2 \leq p<\infty \\
\left\|u-u_{h}\right\|_{0, \infty} & \lesssim h^{2}|\ln h| & \text { if } u \in W^{2, \infty} .
\end{array}
$$

Suppose that $T^{h_{j}}, 1 \leq j \leq J$, is a nested quasi-uniform triangulation of $\Omega$ and the corresponding linear conforming finite element space is defined by

$$
V_{j}=\left\{v \in C(\bar{\Omega}),\left.v\right|_{e} \in P_{1}, \forall e \subset T^{h_{j}}, v=0 \text { on } \partial \Omega\right\} .
$$

Since $T^{h_{j}}$ is nested, we have

$$
V_{0} \subset V_{1} \subset \cdots \subset V_{J} \subset H_{0}^{1}(\Omega) .
$$

For simplicity we assume that

$$
h_{j} \equiv 2^{-j} h_{0} .
$$

The main objective of this work is to propose a multilevel successive iteration method for solving nonlinear elliptic equations. Although the problem under investigation is nonlinear, at each fixed level it is linear. Having this in mind, we will carry out some analysis for the two-grid iterative algorithm and the multilevel linearization methods in this section. 
2.1. A two-grid analysis. Let us consider the linear model problem to get some intuition:

$$
(\nabla u, \nabla v)=(f, v) \quad \forall v \in V=H_{0}^{1}(\Omega),
$$

and the corresponding finite element approximation

$$
\left(\nabla u_{j}, \nabla v\right)=(f, v) \quad \forall v \in V_{j} .
$$

It is easy to see that

$$
\left(\nabla\left(u_{j}-u_{i}\right), \nabla v\right)=0, \quad j \geq i, \quad \forall v \in V_{i} .
$$

This implies that the finite element solution $u_{i}$ approximating $u$ can also be regarded as a finite element approximation to $u_{j}, j \geq i$. The increment $u_{j}-u_{i}$ is orthogonal to the subspace $V_{i}$, indicating that it is of high frequency on the coarser grid $T^{h_{i}}$. One then expects to obtain the increment by some simple smoothing iteration.

Now we turn to the nonlinear equation (2.8). Suppose that we have the exact finite element solution $u_{h_{i}}$ on the level $i$ and want to find an approximation $u_{j}$ of the finite element solution $u_{h_{j}}$ on the level $j, j>i$. It follows from (2.10) that if $u$ is the solution of (2.8) and $w$ is an approximation of $u$, then

$$
\begin{aligned}
A^{\prime}(w ; u, v) & =A^{\prime}(w ; w, v)+A(u, v)-A(w, v)-R(w, u, v) \\
& =A^{\prime}(w ; w, v)+(f, v)-A(w, v)-R(w, u, v) .
\end{aligned}
$$

It is known that $R(w, u, v)$ is a higher order term in $H^{1}(\Omega)$ and may be neglected. This will lead to the following linearized equation:

$$
A^{\prime}\left(u_{h_{i}} ; u_{j}, v\right)=A^{\prime}\left(u_{h_{i}} ; u_{h_{i}}, v\right)+(f, v)-A\left(u_{h_{i}}, v\right) \quad \forall v \in V_{j} .
$$

The solution $u_{j}$ of the above problem is an approximation of $u_{h_{j}}$. This describes a standard two-grid method of 24]. It is easy to see from (2.21) and (2.10) that

$$
A^{\prime}\left(u_{h_{i}} ; u_{j}-u_{h_{j}}, v\right)=R\left(u_{h_{i}}, u_{h_{j}}, v\right) \quad \forall v \in V_{j},
$$

which implies that

$$
\left\|u_{j}-u_{h_{j}}\right\|_{1,2} \lesssim h_{i}^{2}
$$

So the discrete accuracy is guaranteed.

\section{Two-grid iteration algorithm.}

(1) Solve the finite element equation (2.9) for $u_{h_{i}}$ on the level $i$.

(2) Set $u_{j, 0}=u_{h_{i}}$, and solve (2.21) by executing $m_{j}$ smoothing steps on the level $j$. Let $I_{j, m_{j}} u_{j, 0}$ be the output after $m_{j}$ steps of iteration: $u_{j, m_{j}}=$ $I_{j, m_{j}} u_{j, 0}$.

We assume that the error propagation operator $S_{j, m_{j}}: V_{j} \rightarrow V_{j}$ is a linear mapping:

$$
u_{j}-I_{j, m_{j}} u_{j, 0}=S_{j, m_{j}}\left(u_{j}-u_{j, 0}\right) .
$$

We say an iteration is a smoother if it admits the following properties:

$$
\begin{array}{rlrl}
\left\|S_{j, m_{j}} v_{j}\right\|_{a} & \lesssim \frac{h_{j}^{-1}}{m_{j}^{\gamma}}\left\|v_{j}\right\|_{0} & & \forall v_{j} \in V_{j}, \\
\left\|S_{j, m_{j}} v_{j}\right\|_{a} \leq\left\|v_{j}\right\|_{a} & \forall v_{j} \in V_{j},
\end{array}
$$

for some constant $0<\gamma \leq 1$, where $\|\cdot\|_{a}$ is the $a$-norm (energy norm) corresponding to the linear system we wish to solve. It is shown that the Gauss-Seidel iteration, SOR, the Richardson iteration, and the damped Jacobi iteration are all smoothers 
with the constant $\gamma=\frac{1}{2}$; see, e.g., [12, 26]. Moreover, Shaidurov [18] and Bornemann and Deuflhard [2] proved that the CG method behaves like a linear smoother with $\gamma=1$.

The following result gives an error bound for the two-grid iteration algorithm.

Assertion 2.1. Let $u_{j}$ be the exact solution of the linearized equation (2.21) and $u_{j, m_{j}}$ be an approximation of $u_{j}$ defined by the above two-grid iteration algorithm; namely, $u_{j, m_{j}}=I_{j, m_{j}} u_{j, 0}$. If the iteration used is a smoother, then

$$
\left\|u_{j}-u_{j, m_{j}}\right\|_{1} \lesssim \frac{2^{2(j-i)}}{m_{j}^{\gamma}} h_{j}
$$

where $0<\gamma \leq 1$ is defined by 2.24).

Proof. It follows from (2.23)-(2.25) and (2.16) that

$$
\begin{aligned}
\left\|u_{j}-u_{j, m_{j}}\right\|_{1} & \lesssim \frac{h_{j}^{-1}}{m_{j}^{\gamma}}\left\|u_{j}-u_{i}\right\|_{0} \\
& =\frac{h_{j}^{-1}}{m_{j}^{\gamma}}\left\|u_{j}-u+u-u_{i}\right\|_{0} \\
& \lesssim \frac{h_{j}^{-1}}{m_{j}^{\gamma}}\left\|u-u_{i}\right\|_{0} \\
& \lesssim \frac{2^{2(j-i)}}{m_{j}^{\gamma}} h_{j} .
\end{aligned}
$$

This establishes the desired estimate.

The above result shows that to achieve the discretization accuracy on the level $j$ it is sufficient to do $m_{j}$ iterations, with

$$
m_{j} \equiv 2^{\frac{2}{\gamma}(j-i)}
$$

We can see that this $m_{j}$ depends only on the difference $j-i$, and the result is valid for any spatial dimensions.

If we choose $i=j-1$, then only a fixed number of iterations are needed, i.e., $m \equiv 2^{\frac{2}{\gamma}}$, which is independent of the level and the mesh size. However, if we want to iterate the solution on the level $i$ to achieve the discretization accuracy on the level $J$, then the number of iterations required is $m_{i} \equiv 2^{\frac{2}{\gamma}(J-i)}$.

The generalization of the two-grid algorithm to the multilevel gives the cascadic multigrid iteration scheme. The key idea in the generalization is to use the two-grid technique recursively.

2.2. Multilevel linearization method. Similarly to the projective Newton method [23, 16, 22], we can derive the following multilevel linearization algorithm for solving the nonlinear equation (2.8).

Multilevel linearization algorithm. [1, 13, 24]

(1) Find $u_{0} \in V_{0}$ such that $A\left(u_{0}, v\right)=(f, v) \quad \forall v \in V_{0}$.

(2) For $j=1,2, \ldots, J$, find $u_{j} \in V_{j}$ such that

$$
A^{\prime}\left(u_{j-1} ; u_{j}, v\right)=A^{\prime}\left(u_{j-1} ; u_{j-1}, v\right)+(f, v)-A\left(u_{j-1}, v\right) \quad \forall v \in V_{j}
$$


Let $u_{h_{j}}$ be the solution of (2.9) in $V_{j}$. From (2.26) and (2.10) we have

$$
\begin{aligned}
A^{\prime}\left(u_{j-1} ; u_{j}-u_{j-1}, v\right) & =(f, v)-A\left(u_{j-1}, v\right) \\
& =A\left(u_{h_{j}}, v\right)-A\left(u_{j-1}, v\right) \\
& =A^{\prime}\left(u_{j-1} ; u_{h_{j}}-u_{j-1}, v\right)+R\left(u_{j-1}, u_{h_{j}}, v\right),
\end{aligned}
$$

which gives

$$
A^{\prime}\left(u_{j-1} ; u_{j}-u_{h_{j}}, v\right)=R\left(u_{j-1}, u_{h_{j}}, v\right) \quad \forall v \in V_{j} .
$$

We now prove some superconvergence properties for the error between $u_{h_{j}}$, the exact solution of (2.9), and $u_{j}$, the approximation of $u_{h_{j}}$ given by (2.26).

Assertion 2.2. Assume $h_{0} \ll 1$, and let $u$ be the solution of (2.8). The following results hold:

- If $u \in W^{2, \infty}(\Omega)$, then

$$
\left\|u_{j}-u_{h_{j}}\right\|_{1, \infty} \lesssim h_{j}^{2}\left|\ln h_{j}\right| .
$$

- If $u \in W^{2,4}(\Omega)$, then

$$
\left\|u_{j}-u_{h_{j}}\right\|_{1,2} \lesssim h_{j}^{2} .
$$

Proof. By Lemma 2.1 we may assume that

$$
\left\|u_{h_{j}}-u_{h_{j-1}}\right\|_{1, \infty} \leq C_{2} h_{j} .
$$

Moreover, we may also choose $h_{0} \ll 1$ such that $u_{h_{j}} \in \mathcal{B}_{K / 2}$; namely,

$$
\left\|u-u_{h_{j}}\right\|_{1, \infty} \leq \frac{K}{2},
$$

where the constant $K$ is the same as in (2.4). It is known that the finite element approximation $G_{h}$ of the regularized Green function of derivative type related to the bilinear form $A^{\prime}(w ; \phi, v)$ satisfies the following inequality [7, 17]:

$$
\left\|G_{h}\right\|_{1,1} \leq C_{0}|\ln h| .
$$

Let $C_{1}=M C_{0} / \alpha_{0}$ and $C=4 C_{1} C_{2}^{2}$. Choose $h_{0} \ll 1$ such that

$$
2 C h_{0}\left|\ln h_{0}\right| \leq C_{2} \text {. }
$$

Now we prove (2.28) by induction. For $j=0$, (2.28) holds since $u_{0}=u_{h_{0}}$. Under the assumptions

$$
u_{j-1} \in \mathcal{B}_{K} \quad \text { and } \quad\left\|u_{j-1}-u_{h_{j-1}}\right\|_{1, \infty} \leq C h_{j-1}^{2}\left|\ln h_{j-1}\right|,
$$

we will show that (2.33) is also true when the index $j-1$ is replaced by $j$. Taking $v=G_{h}$ in (2.27), the ellipticity (2.4) and the boundedness (2.7) yield

$$
\begin{aligned}
\left\|u_{j}-u_{h_{j}}\right\|_{1, \infty} & \leq C_{1}\left\|u_{h_{j}}-u_{j-1}\right\|_{1, \infty}^{2}\left|\ln h_{j}\right| \\
& \leq 2 C_{1}\left(\left\|u_{h_{j}}-u_{h_{j-1}}\right\|_{1, \infty}^{2}+\left\|u_{h_{j-1}}-u_{j-1}\right\|_{1, \infty}^{2}\right)\left|\ln h_{j}\right| \\
& \leq 2 C_{1}\left(C_{2}^{2} h_{j}^{2}+C^{2} h_{j-1}^{4}\left|\ln h_{j-1}\right|^{2}\right)\left|\ln h_{j}\right| \\
& \leq 2 C_{1}\left(C_{2}^{2}+4 C^{2} h_{j-1}^{2}\left|\ln h_{j-1}\right|^{2}\right) h_{j}^{2}\left|\ln h_{j}\right| \\
& \leq 4 C_{1} C_{2}^{2} h_{j}^{2}\left|\ln h_{j}\right| \\
& \leq C h_{j}^{2}\left|\ln h_{j}\right|
\end{aligned}
$$


and

$$
\begin{aligned}
\left\|u_{j}-u\right\|_{1, \infty} & \leq\left\|u_{j}-u_{h_{j}}\right\|_{1, \infty}+\left\|u_{h_{j}}-u\right\| \\
& \leq C h_{j}^{2}\left|\ln h_{j}\right|+\frac{K}{2} \leq K .
\end{aligned}
$$

This completes the induction proof for (2.28). We will now prove (2.29). Let the constant $C_{2}$ in (2.30) also be the bounding constant for the $W^{1,4}$ norm, i.e.,

$$
\left\|u_{h_{j}}-u_{h_{j-1}}\right\|_{1,4} \leq C_{2} h_{j} .
$$

Let $C_{3}$ be the bounding constant in the inverse estimate of the finite element spaces, i.e.,

$$
\|v\|_{1, p} \leq C_{3} h_{j}^{\frac{d}{p}-\frac{d}{q}}\|v\|_{1, q} \quad \forall v \in V_{j} .
$$

Assume $h_{0} \ll 1$ such that $2 C C_{3} h_{0}^{1-\frac{d}{4}} \leq C_{2}$. Again we use induction to prove (2.29). For $j=0$, (2.29) holds since $u_{0}=u_{h_{0}}$. Assume

$$
u_{j-1} \in \mathcal{B}_{k} \quad \text { and } \quad\left\|u_{j-1}-u_{h_{j-1}}\right\|_{1,2} \leq C h_{j-1}^{2} ;
$$

we will show that (2.37) is also true when the index $j-1$ is replaced by $j$. Similarly to the derivation of (2.34), we have

$$
\begin{aligned}
\left\|u_{j}-u_{h_{j}}\right\|_{1,2} & \leq C_{1}\left\|u_{h_{j}}-u_{j-1}\right\|_{1,4}^{2} \\
& \leq 2 C_{1}\left(\left\|u_{h_{j}}-u_{h_{j-1}}\right\|_{1,4}^{2}+\left\|u_{h_{j-1}}-u_{j-1}\right\|_{1,4}^{2}\right) \\
& \leq 2 C_{1}\left(C_{2}^{2} h_{j}^{2}+C_{3}^{2} h_{j-1}^{-\frac{d}{2}}\left\|u_{h_{j-1}}-u_{j-1}\right\|_{1,2}^{2}\right) \\
& \leq 2 C_{1}\left(C_{2}^{2}+4 C^{2} C_{3}^{2} h_{j-1}^{2\left(1-\frac{d}{4}\right)}\right) h_{j}^{2} \\
& \leq 4 C_{1} C_{2}^{2} h_{j}^{2} \leq C h_{j}^{2},
\end{aligned}
$$

and

$$
\begin{aligned}
\left\|u-u_{j}\right\|_{1, \infty} & \leq\left\|u-u_{h_{j}}\right\|_{1, \infty}+C_{3} h_{j}^{-\frac{d}{2}}\left\|u_{h_{j}}-u_{j}\right\|_{1,2} \\
& \leq \frac{K}{2}+C_{3} C h_{j}^{2-\frac{d}{2}} \leq K .
\end{aligned}
$$

This completes the proof.

The superconvergence estimates (2.28) and (2.29) appear to be new. Similar results for the derivatives were obtained by $\mathrm{Xu}$ [24] and Bank [1]. The following corollary is a direct consequence of the above assertion.

Corollary 2.1. Assume $h_{0} \ll 1$ and let $u$ be the solution of (2.8).

- If $u \in W^{2, \infty}(\Omega)$, then

$$
\begin{aligned}
\left\|u-u_{j}\right\|_{1, \infty} & \lesssim h_{j}, \\
\left\|u-u_{j}\right\|_{0, \infty} & \lesssim h_{j}^{2}\left|\ln h_{j}\right| .
\end{aligned}
$$

- Furthermore, if $u \in W^{2,4}(\Omega)$, then

$$
\begin{aligned}
\left\|u-u_{j}\right\|_{1,4} & \lesssim h_{j}, \\
\left\|u-u_{j}\right\|_{0,2} & \lesssim h_{j}^{2} .
\end{aligned}
$$


2.3. Inexact multilevel linearization method. In the multilevel linearization method stated above, one must compute $u_{j}$ exactly by using (2.26) with the initial value $u_{j-1}$. However, in practice, $u_{j}$ is only approximated up to some accuracy by using approximation methods or the so-called inexact solvers. In this subsection we analyze the influence of the inexact solver on each grid level to the final grid solution. The error estimates under both the energy norm and the $L_{2}$ norm will be obtained. To prove the $L_{2}$ norm estimate, a duality argument for nonlinear problems will be developed. We concentrate only on the 2-D case.

Suppose we use an inexact solver in the multilevel linearization method at the level $j$ and obtain the approximation $u_{j}^{*}, j=1, \ldots, J$. Let $U_{j}$ be the exact solution of the linearized equation (2.26) with the initial value $u_{j-1}^{*}, j \geq 1$. Denote by $e_{j}$ the error of this solver on the level $j$, i.e.,

$$
u_{j}^{*}=U_{j}+e_{j} .
$$

Then the inexact multilevel linearization algorithm is as follows.

\section{Inexact Multilevel Linearization Algorithm.}

(1) Let $U_{0}=u_{h_{0}} \in V_{0}$ be the solution of $A\left(U_{0}, v\right)=(f, v) \quad \forall v \in V_{0}$.

(2) For $j=1,2, \ldots, J$, find $U_{j} \in V_{j}$ such that

$$
A^{\prime}\left(u_{j-1}^{*} ; U_{j}, v\right)=A^{\prime}\left(u_{j-1}^{*} ; u_{j-1}^{*}, v\right)+(f, v)-A\left(u_{j-1}^{*}, v\right) \quad \forall v \in V_{j} .
$$

Let $u_{h_{j}}$ be the solution of (2.9) in $V_{j}$. Similarly to (2.22), we have

$$
\begin{aligned}
A^{\prime}\left(u_{j-1}^{*} ; U_{j}-u_{j-1}^{*}, v\right) & =(f, v)-A\left(u_{j-1}^{*}, v\right) \\
& =A\left(u_{h_{j}}, v\right)-A\left(u_{j-1}^{*}, v\right) \\
& =A^{\prime}\left(u_{j-1}^{*} ; u_{h_{j}}-u_{j-1}^{*}, v\right)+R\left(u_{j-1}^{*}, u_{h_{j}}, v\right) .
\end{aligned}
$$

Hence

$$
A^{\prime}\left(u_{j-1}^{*} ; U_{j}-u_{h_{j}}, v\right)=R\left(u_{j-1}^{*}, u_{h_{j}}, v\right) \quad \forall v \in V_{j} .
$$

Now we turn to the error analysis for the solution $U_{j}$ of 2.45) given by the inexact multilevel linearization algorithm and the exact nonlinear solution $u_{h_{j}}$ on the level $j$.

Assertion 2.3. Assume $h_{0} \ll 1$, and let $u$ be the solution of (2.8). If $u \in W^{2,4}(\Omega)$ and the error $e_{j}$, defined by (2.44), satisfies

$$
\left\|e_{j}\right\|_{1} \leq \delta h_{j}, \quad\left\|e_{j}\right\|_{1} \leq C_{e}\left\|e_{j+1}\right\|_{1}, \quad j=0,1, \ldots, J-1,
$$

then

$$
\left\|U_{j}-u_{h_{j}}\right\|_{1,2} \lesssim h_{j}^{2}+\left\|e_{j}\right\|_{1}, \quad j \leq J .
$$

Furthermore, if $\Omega$ is smooth or convex, then

$$
\left\|U_{j}-u_{h_{j}}\right\|_{0,2} \lesssim h_{j}^{2}+\left\|e_{j}\right\|_{1}^{2}\left|\ln h_{j}\right|^{\frac{1}{2}}, \quad j \leq J .
$$

Proof. We prove the above results by induction again. If $u_{j-1}^{*}$ is in the neighborhood of $u$, using (2.11) and (2.46), we have

$$
\begin{aligned}
\left\|U_{j}-u_{h_{j}}\right\|_{1,2} & \leq C_{1}\left\|u_{h_{j}}-u_{j-1}^{*}\right\|_{1,4}^{2} \\
& \leq 2 C_{1}\left(\left\|u_{h_{j}}-u_{h_{j-1}}\right\|_{1,4}^{2}+\left\|u_{h_{j-1}}-u_{j-1}^{*}\right\|_{1,4}^{2}\right) \\
& \leq 2 C_{1}\left\{C_{2}^{2} h_{j}^{2}+2 C_{3}^{2} h_{j-1}^{-1}\left(\left\|u_{h_{j-1}}-U_{j-1}\right\|_{1,2}^{2}+\left\|e_{j-1}\right\|_{1,2}^{2}\right)\right\},
\end{aligned}
$$


where $C_{2}$ and $C_{3}$ are constants defined in (2.35) and (2.36), respectively. Let $C=4 C_{1} C_{2}^{2}$. Suppose $h_{0}$ is small enough so that

$$
\left\|u-u_{h_{j}}\right\|_{1, \infty} \leq K / 2, \quad 64 C_{1} C_{3}^{2} h_{0} \leq 1, \quad C C_{3} h_{0} \leq K / 4 .
$$

Suppose $\delta \ll 1$ is such that

$$
C_{3} C_{e} \delta \leq K / 4, \quad 12 C_{1} C_{3}^{2} C_{e}^{2} \delta \leq 1 .
$$

For $j=0$ we have $U_{0}-u_{h_{0}}=0$, so the estimate (2.47) is valid. Furthermore, by (2.50) and (2.51) we have

$$
\begin{aligned}
\left\|u-u_{0}^{*}\right\|_{1, \infty} & \leq\left\|u-u_{h_{0}}\right\|_{1, \infty}+\left\|e_{0}\right\|_{1, \infty} \\
& \leq K / 2+C_{3} \delta \leq K,
\end{aligned}
$$

which implies that $u_{0}^{*} \in \mathcal{B}_{K}$. Suppose (2.47) holds for $j \leq i-1$, i.e.,

$$
\left\|U_{j}-u_{h_{j}}\right\|_{1} \leq C h_{j}^{2}+\left\|e_{j}\right\|_{1}^{2}, \quad j<i .
$$

It can be verified that $u_{i-1}^{*} \in \mathcal{B}_{K}$, and

$$
\begin{aligned}
\left\|u-u_{i-1}^{*}\right\|_{1, \infty} & \leq\left\|u-U_{i-1}\right\|_{1, \infty}+\left\|e_{i-1}\right\|_{1, \infty} \\
& \leq K / 2+C_{3} C_{e} \delta \leq K .
\end{aligned}
$$

The assumption (2.53), together with (2.49)-(2.51), yields

$$
\begin{aligned}
\left\|U_{i}-u_{h_{i}}\right\|_{1,2} & \leq 2 C_{1}\left(C_{2}^{2}+16 C C_{3}^{2} h_{i}\right) h_{i}^{2}+12 C_{1} C_{3}^{2} C_{e}^{2} \delta\left\|e_{i}\right\|_{1} \\
& \leq C h_{i}^{2}+\left\|e_{i}\right\|_{1},
\end{aligned}
$$

which means that (2.47) is valid for $j=i$. It remains to verify that $U_{i}$ is still in the neighborhood of $u$, i.e., $U_{i} \in \mathcal{B}_{k}$, in order to complete the proof of (2.47). In fact, using (2.50), (2.51) and the inverse estimates gives

$$
\begin{aligned}
\left\|u-U_{i}\right\|_{1, \infty} & \leq\left\|u-u_{h_{i}}\right\|_{1, \infty}+C_{3} h_{i}^{-1}\left\|u_{h_{i}}-U_{i}\right\|_{1} \\
& \leq \frac{K}{2}+C_{3}\left(C h_{i}+\delta\right) \leq K .
\end{aligned}
$$

Finally, we prove the optimal $L_{2}$ error estimate for $u_{j}$ generated by the inexact multilevel linearization algorithm. We use a duality argument. Construct the following auxiliary problem: Find $w \in H_{0}^{1}(\Omega)$ such that

$$
A^{\prime}\left(u_{j-1}^{*} ; v, w\right)=\left(v, U_{j}-u_{h_{j}}\right) \quad \forall v \in H_{0}^{1}(\Omega) .
$$

Let $w_{j} \in V_{j}$ be the finite element approximation of $w$ in $V_{j}$, and set $v=U_{j}-u_{h_{j}}$ in (2.57). It follows from (2.46), (2.11) and (2.7) that

$$
\begin{aligned}
\left\|U_{j}-u_{h_{j}}\right\|_{0,2}^{2} & =A^{\prime}\left(u_{j-1}^{*} ; U_{j}-u_{h_{j}}, w\right) \\
& =A^{\prime}\left(u_{j-1}^{*} ; U_{j}-u_{h_{j}}, w-w_{j}\right)+A^{\prime}\left(u_{j-1}^{*} ; U_{j}-u_{h_{j}}, w_{j}\right) \\
& =A^{\prime}\left(u_{j-1}^{*} ; U_{j}-u_{h_{j}}, w-w_{j}\right)+R\left(u_{j-1}^{*}, u_{h_{j}}, w_{j}\right) \\
& \lesssim\left\|U_{j}-u_{h_{j}}\right\|_{1}\left\|w-w_{j}\right\|_{1}+\left\|u_{h_{j-1}}-u_{h_{j}}\right\|_{1,4}^{2}\left\|w_{j}\right\|_{1} \\
& \quad+\left(\left\|U_{j-1}-u_{h_{j-1}}\right\|_{1}^{2}+\left\|e_{j-1}\right\|_{1}^{2}\right)\left\|w_{j}\right\|_{1, \infty} \\
& \lesssim\left(h_{j}^{2}+\left\|e_{j}\right\|_{1}^{2}\left|\ln h_{j}\right|^{\frac{1}{2}}\right)\|w\|_{2} .
\end{aligned}
$$

In the last inequality we have used the fact that $\left\|w_{j}\right\|_{1, \infty} \lesssim\left|\ln h_{j}\right|^{\frac{1}{2}}\|w\|_{2}$, which can be obtained by using the inverse estimates, the $W^{1, p}$-stability property and the embedding theorem. Hence, applying standard a priori estimates yields (2.48). 


\section{Multilevel SuCCESSive iteration METHOD}

Based on the preparations in the last section, we propose the following multilevel successive iterative algorithm for solving the nonlinear equation (2.9).

\section{Multilevel Successive Iteration Algorithm.}

(1) Solve the nonlinear problem $A\left(u_{h_{0}}, v\right)=(f, v) \quad \forall v \in V_{0}$ by an appropriate solver to obtain $u_{0}^{*}$ with the accuracy $\delta h_{0}$ and $h_{0}^{2}$ in the $H^{1}$-norm and $L_{2}$-norm, respectively.

(2) For $j=1,2, \ldots, J$, let $I_{j, m_{j}}$ denote $m_{j}$ basic smoothing iterations on the level $j$ with initial data $u_{j-1}^{*}$ for solving the linearized equation

$$
A^{\prime}\left(u_{j-1}^{*} ; U_{j}, v\right)=A^{\prime}\left(u_{j-1}^{*} ; u_{j-1}^{*}, v\right)+(f, v)-A\left(u_{j-1}^{*}, v\right) \quad \forall v \in V_{j},
$$

and set $u_{j}^{*}=I_{j, m_{j}} u_{j-1}^{*}$.

Now we give the error analysis for the exact solution $U_{j}$ and its approximation $u_{j}^{*}$ in the above algorithm. Let $u_{j}^{*}=U_{j}-e_{j}$. Then

$$
\begin{aligned}
e_{j} & =U_{j}-u_{j}^{*}=S_{j, m_{j}}\left(U_{j}-u_{j-1}^{*}\right) \\
& =S_{j, m_{j}}\left(U_{j}-U_{j-1}\right)+S_{j, m_{j}} e_{j-1} .
\end{aligned}
$$

Define the level-dependent norm

$$
\|v\|_{a, j}^{2}=A^{\prime}\left(u_{j-1}^{*} ; v, v\right) \quad \forall v \in V_{j}, j \geq 1 .
$$

Lemma 3.1. Assume $h_{0} \ll 1$, and let $u$ be the solution of (2.8). If $u \in W^{2,4}(\Omega)$ and the error $e_{j}$, defined by (2.44), satisfies

$$
\left\|e_{j}\right\|_{1} \leq \delta h_{j}, \quad\left\|e_{j}\right\|_{1} \leq C_{e}\left\|e_{j+1}\right\|_{1}, \quad j=0,1, \ldots, J-1,
$$

then for any $v \in V_{j}, j \geq 2$,

$$
\|v\|_{a, j}^{2} \leq\left(1+C h_{j}^{\frac{1}{2}}+C\left\|e_{j-1}\right\|_{1, \infty}+C\left\|e_{j-2}\right\|_{1, \infty}\right)\|v\|_{a, j-1}^{2}
$$

where $C$ is a constant independent of the mesh size $h$ and the level $j$.

Proof. It follows from the definitions (2.6) and (3.2) that

$$
\begin{aligned}
\|v\|_{a, j}^{2} & =A^{\prime}\left(u_{j-1}^{*} ; v, v\right) \\
& =\|v\|_{a, j-1}^{2}+A^{\prime}\left(u_{j-1}^{*} ; v, v\right)-A^{\prime}\left(u_{j-2}^{*} ; v, v\right) \\
& \leq\|v\|_{a, j-1}^{2}+C\left|u_{j-1}^{*}-u_{j-2}^{*}\right|_{1, \infty}\|v\|_{1}^{2} .
\end{aligned}
$$

Using the inverse estimate (2.36) and Lemma 2.1, we obtain

$$
\begin{aligned}
\mid u_{h_{j-1}}-u_{h_{j-2} \mid 1, \infty} & \lesssim h_{j-1}^{-\frac{1}{2}}\left\|u_{h_{j-1}}-u_{h_{j-2}}\right\|_{1,4} \\
& \lesssim h_{j}^{\frac{1}{2}}
\end{aligned}
$$

If we use estimates (3.4) and (3.5), then Assertion 2.3 together with the identities

$$
\begin{aligned}
& u_{j-1}^{*}-u_{j-2}^{*}=U_{j-1}-U_{j-2}-e_{j-1}-e_{j-2}, \\
& U_{j-1}-U_{j-2}=\left(U_{j-1}-u_{h_{j-1}}\right)+\left(u_{h_{j-1}}-u_{h_{j-2}}\right)+\left(u_{h_{j-2}}-U_{j-2}\right),
\end{aligned}
$$

yields the desired inequality (3.3). 
Theorem 3.1. Assume that $d=2, h_{0} \ll 1, u \in W^{2,4}(\Omega)$ and $\Omega$ is smooth or convex. If we choose the number of iterations as $m_{j}=m \tilde{m}_{j}$ with

$$
\tilde{m}_{j}= \begin{cases}{\left[J^{2} 2^{2(J-j)}\right]+1} & \text { if } \gamma=\frac{1}{2}, \\ {\left[J^{\frac{1}{2}} 2^{\beta(J-j)}\right]+1,1<\beta<2,} & \text { if } \gamma=1,\end{cases}
$$

where $m$ is a proper constant, then the multilevel successive iteration algorithm admits the error bound

$$
\left\|e_{j}\right\|_{a, j} \lesssim h_{J}, \quad j=1,2, \ldots, J
$$

and the total number of operations is bounded by

$$
W_{J} \lesssim \begin{cases}J^{3} N_{J}, & \gamma=\frac{1}{2}, \\ \sqrt{J} N_{J}, & \gamma=1 .\end{cases}
$$

Proof. Without loss of generality, we may choose $h_{0} \ll 1$ as in (2.31) such that

$$
\left\|u-U_{j}\right\|_{1, \infty} \leq K / 2 .
$$

We establish the error bound (3.7) by induction. For $j=0$, the result is obvious. Suppose that

$$
\left\|e_{j}\right\|_{a}<h_{J} K / 2
$$

for $j \leq i-1$. Then

$$
\begin{array}{cl}
\left\|u_{j}^{*}-u\right\|_{1, \infty} \leq K, & j=1, \ldots, i-1, \\
\left\|e_{j}\right\|_{1, \infty} \lesssim h_{j}^{-1}\left\|e_{j}\right\|_{1,2} \lesssim \frac{h_{J}}{h_{j}} \lesssim 2^{j-J}, & j=1, \ldots, i-1 .
\end{array}
$$

By using Lemma 3.1 the smoothing property (2.25), and (3.12), we have

$$
\begin{aligned}
\left\|e_{i}\right\|_{a, i} & \leq\left\|S_{i, m_{i}}\left(U_{i}-U_{i-1}\right)\right\|_{a, i}+\left\|S_{i, m_{i}} e_{i-1}\right\|_{a, i} \\
& \leq\left\|S_{i, m_{i}}\left(U_{i}-U_{i-1}\right)\right\|_{a, i}+\left\|e_{i-1}\right\|_{a, i} \\
& \leq\left\|S_{i, m_{i}}\left(U_{i}-U_{i-1}\right)\right\|_{a, i}+\left(1+C 2^{j-1-J}+C h_{i-1}^{\frac{1}{2}}\right)\left\|e_{i-1}\right\|_{a, i-1} \\
& \leq \sum_{j=1}^{i} \prod_{l=j}^{i-1}\left(1+C 2^{l-J}+C h_{l}^{\frac{1}{2}}\right)\left\|S_{j, m_{j}}\left(U_{j}-U_{j-1}\right)\right\|_{a, j} \\
& \leq \tilde{C}_{1} \sum_{j=1}^{i}\left\|S_{j, m_{j}}\left(U_{j}-U_{j-1}\right)\right\|_{a, j} .
\end{aligned}
$$

Furthermore, it follows from the smoothing property (2.24) and the superconvergence result 2.41) that

$$
\begin{aligned}
\left\|e_{i}\right\|_{a, i} & \leq \frac{\tilde{C}_{1}}{m^{\gamma}} \sum_{j=1}^{i}\left(\frac{h_{j}}{\tilde{m}_{j}^{\gamma}}+\frac{h_{j}^{-1} h_{J}^{2}\left|\ln h_{j}\right|^{\frac{1}{2}}}{\tilde{m}_{j}^{\gamma}}\right) \\
& \leq \frac{\tilde{C}_{1} \tilde{C}_{2}}{m^{\gamma}} h_{J}
\end{aligned}
$$

with

$$
\tilde{C}_{2}=\left(\sum_{j=1}^{J} \frac{2^{J-j}}{\tilde{m}_{j}^{\gamma}}+\sum_{j=1}^{J} \frac{J^{\frac{1}{2}} 2^{j-J}}{\tilde{m}_{j}^{\gamma}}\right)
$$


where the iteration number $\tilde{m}_{j}$ is given by (3.6). Hence

$$
\left\|e_{i}\right\|_{a, i} \leq \frac{K}{2} h_{J}
$$

provided $m$ is sufficiently large. So (3.7) holds for $j=i$, as long as $u_{i}^{*}$ is still in the neighborhood of $u$. The latter requirement can be verified by the following observations:

$$
\begin{aligned}
\left\|u_{i}^{*}-u\right\|_{1, \infty} & \leq\left\|u_{i}^{*}-U_{i}\right\|_{1, \infty}+\left\|U_{i}-u\right\|_{1, \infty} \\
& \leq \frac{K}{2}+\frac{K}{2} \leq K .
\end{aligned}
$$

Therefore, (3.7) is proved. Finally, we will estimate the total number of operations (3.8). For $\gamma=1 / 2$,

$$
\begin{aligned}
W_{J} & =\sum_{j=1}^{J} m_{j} N_{j} \lesssim \sum_{j=1}^{J} J^{2} 2^{2(J-j)} 2^{2 j} N_{0} \\
& \lesssim J^{3} N_{J}
\end{aligned}
$$

while for $\gamma=1$,

$$
\begin{aligned}
W_{J} & =\sum_{j=1}^{J} m_{j} N_{j} \lesssim \sum_{j=1}^{J} J^{\frac{1}{2}} 2^{\beta(J-j)} 2^{2 j} N_{0} \\
& \lesssim J^{\frac{1}{2}} N_{J} \sum_{j=1}^{J} 2^{(\beta-2)(J-j)} \\
& \lesssim J^{\frac{1}{2}} N_{J}
\end{aligned}
$$

This completes the proof of this theorem.

Theorem 3.1 shows that for a fixed grid level $J$ the complexity of the proposed multilevel successive iteration algorithm is optimal, and is proportional to the total number of unknowns. For an arbitrary grid level the algorithm is quasi-optimal, since $J \equiv \ln N_{J}$ asymptotically.

We close this section with the following observations:

- First, we only considered the error estimate in the $H^{1}$-norm in this paper. However, it is easy to see that for a fixed level $J$, we can obtain the optimal convergence rate in any norm, provided that the operation number is proportional to the number of unknowns on the final grid. On the other hand, if the mesh level $J$ is arbitrary as in the case of cascadic iterations, then only the $H^{1}$-norm estimate can be kept optimal.

- Although our analysis for nonlinear equations is given only for the 2-D case, it can be extended to 3-D problems when the mesh level $J$ is fixed. However, if the mesh level $J$ is arbitrary, the situation is more complicated. In this case, the cascadic multigrid method only achieves the optimal accuracy in the energy norm, and as a result the most difficult step is to verify whether the iterative approximation $u_{j}^{*}$ remains in the neighborhood of $u$ uniformly in the $W^{1, \infty}$-norm. Unforturnately, this is not the case for strongly nonlinear equations in 3-D. Therefore, it seems difficult to extend the 2-D analysis presented in this section to deal with 3-D problems when $J$ is not fixed. This remains one of our further research topics. 


\section{ACKNOWLEDGMENTS}

We would like to thank an anonymous referee for helpful suggestions. The research of Y. Huang and Z. Shi was supported in part by special funds for Major State Basic Research Projects of China, and Y. Huang was also supported in part by the National Education Ministry. The research of T. Tang and W. Xue was supported by the FRG grants of the Hong Kong Baptist University and the RGC grants of the Hong Kong Research Grants Council.

\section{REFERENCES}

[1] R. BAnk, Analysis of a multilevel iterative method for nonlinear finite element equations. Math. Comp., 39 (1982), 453-465. MR 83j:65105

[2] F. Bornemann and P. Deuflhard, The cascadic multigrid method for elliptic problems, Numer. Math., 75 (1996), 135-152. MR 98a:65175

[3] F. Bornemann and P. Deuflhard The cascadic multigrid method. Proceedings of the 8th international conference on domain decomposition methods, R.Glowinski, J.Periaux, Z. Shi and O.Widlund eds., Wiley and Sons, 1997, pp. 205-212.

[4] D. Braess and W. Dehmen, A cascade algorithm for the Stokes equations. Numer. Math., 82 (1999), 179-191. MR 2000c:65114

[5] J.H. Bramble, J.E. Pasciak and J. Xu, Parallel multilevel preconditioner. Math. Comp., 55 (1990), 1-22. MR 90k:65170

[6] A. Brandt, Multilevel adaptive solutions to boundary-value problems. Math. Comp., 31 (1977), 333-390. MR 55:4714

[7] C. Chen And Y. Huang, $W^{1, p}$-Stability of finite element method for elliptic problems. Hunan Annals of Mathematics, 6 (1986), 81-89. (Chinese) MR 92e:65144

[8] C. Chen and Y. Huang, High accuracy theory of finite element methods, Hunan Science and Technology Press, 1995. (Chinese)

[9] P. Deuflhard, Cascadic conjugate gradient methods for elliptic partial differential equations. Proceedings of the 7th international conference on domain decomposition methods, eds D. Keyes and J. Xu, AMS, Providence, 1994, pp. 29-42. MR 95i:65006

[10] P. Deuflhard, P. Leinen and H. Yserentant, Concepts of an adaptive hierarchical finite element code. IMPACT 1 (1989), 3-35.

[11] J. Frehse and R. Rannacher, Asymptotic $L_{\infty}$-error estimate for linear finite element approximation of quasilinear boundary value problems. SIAM J. Numer. Anal., 15 (1978), 418-431. MR 58:19224]

[12] W. HackBusch Multi-grid methods and applications. Springer Verlag, Berlin-HeidelbergNew York, 1992. MR 87e:65082

[13] Y. Huang And Y. Chen, A multilevel iterate correction method for solving nonlinear singular problems, Natural Science J of Xiangtan Univ. 16 (1994), 23-26. (Chinese)

[14] H. Huang and G. Liu, Successive refinement method for solving elliptic boundary value problems. Mathematica Numerica Sinica, 2 (1978), 41-52, and 3 (1978), 28-35. (Chinese)

[15] R. Rannacher, On the finite element approximation of general boundary value problems in nonlinear elasticity. Calcolo, 17 (1980), 175-193. MR 82f:73019

[16] R. RANNACHER, On the convergence of the Newton-Raphson method for strongly nonlinear finite element equations. In Nonlinear Computational Mechanics, eds P. Wriggers and W. Wagner, Springer Verlag, 1991.

[17] R. Rannacher And R. Scot, Some optimal error estimates for piecewise linear finite element approximations. Math. Comp., 38 (1982), 437-445. MR 83e:65180

[18] V. Shaidurov, Some estimates of the rate of convergence for the cascadic conjugate gradient method. Comp. Math. Appl., 31 (1996), 161-171.

[19] Z. Shi ANd X. XU, Cascadic multigrid method for elliptic problems, East-West J. Numer. Math., 7 (1999), 199-222. MR 2000h:66185

[20] Z. Shi And X. XU, Cascadic multigrid method for a plate bending problem. East-West J. Numer. Math., 6 (1998), 137-153. MR 99g:73109

[21] Z. Shi And X. Xu, Cascadic multigrid method for parabolic problem. J. Comput. Math., 18 (2000), 551-560. MR 2001e:65151 
[22] G. VAINikKo, Galerkin's perturbation method and the general theory of approximation for nonlinear equations. USSR Comput. Math. and Math. Phys., 7 (1967), 1-41. MR 36:1095

[23] K. Witsch, Projective Newton-Verfahren und Adwendungen auf nicht linear Randwertaufgaben. Numer. Math., 31 (1978/1979), 209-230. MR 80a:65160

[24] J. XU, Two-grid discretization techniques for linear and nonlinear PDEs. SIAM J. Numer. Anal., 33, (1996), 1759-1777. MR 97i:65169

[25] J. Xu, A novel two-grid method for semilinear equations. SIAM J. Sci. Comput., 15 (1994), 231-237. MR 94m:65178

[26] J. Xu, Iterative methods by space decomposition and subspace correction, SIAM Review, 34 (1992), 581-613. MR 93k:65029

[27] H. Yserentant, On the multilevel splitting of finite element spaces. Numer. Math., 49 (1986), 379-412. MR 88d:65068a: MR 88d:65068b

Department of Mathematics and Institute for Computational and Applied Mathematics, Xiangtan University, Xiangtan, Hunan 411105, Peoples Republic of China

E-mail address: huangyq@xtu.edu.cn

Institute of Computational Mathematics, Chinese Academy of Sciences, Beijing, 100080, Peoples Republic of China

E-mail address: shi@lsec.cc.ac.cn

Department of Mathematics, Hong Kong Baptist University, Kowloon Tong, Hong KONG

E-mail address: ttang@math.hkbu.edu.hk

Department of Mathematics, Hong Kong Baptist University, Kowloon Tong, Hong KONG

E-mail address: wmxue@math.hkbu.edu.hk 